\title{
Heart and teeth: old enemies finally reconciled?
}

\author{
O. Leborgne
}

Cardiologist, Independent Hospital

\section{ABSTRACT}

Dealing with cardio-vascular system, two types of complications may happen during oral and dental care. The first one is the risk of focal infection, especially at the endocardium. The second one is the hemorrhagic risk. New guidelines, edited by international societies of cardiology and buccal surgery, have questioned our conventional way of thinking. The purpose of this topic is to decline these recommendations to orthodontics.

\section{KEYWORDS}

Infectious endocarditis, hemorrhagic risk, orthodontics

\section{INTRODUCTION}

The heart and teeth have never had a harmonious relationship. Infectious endocarditis is a life-threatening disease. Recommendations have long been based on expert advice resulting from a limited number of lowincidence randomized trials or meta-analyses. Numerous kinds of oral surgery, including orthodontic bracket placement, have justified the prescription of an antibiotic prophylaxis. Recent protocol updates have decreased the rate of prescription of antibiotics before dental care by $78 \%$. The other risk faced is hemorrhage. Even if few young orthodontic patients benefit from antiaggregation or anticoagulant treatment, the increasing number of adult patients forces us to consider this risk. We therefore describe the management of patients with infections in light of the 2015 recommendations and describe the risk of hemorrhage.

\section{THE INFECTION}

For years, cardiologists have been tracking dental infections in patients who have undergone heart valve surgery. Indeed,
$80 \%$ of cardiac heart valve infections (endocarditis) are of dental origin ${ }^{4}$. This is due to microbial flora, which is abundant in the
Address for correspondence:

Olivier Lweborgne - 11 avenue Jules-Ferry - 35700 Rennes

E-mail: olivier_leb@yahoo.fr
Article received: 15-09-2016. Accepted for publication: 30-09-2016.

This is an Open Access article distributed under the terms of the Creative Commons Attribution License (http://creativecommons.org/licenses/by/4.0), which permits unrestricted use, distribution, and reproduction in any medium, provided the original work is properly cited. 
oral-dental environment, in particular streptococci ${ }^{6}$. In addition, the endocardium is susceptible to infections.

For a long time, before heart valve surgery, "preoperative" assessment ended with a systematic extraction of all infected or possibly infected teeth to eliminate sources of possible infection. The result was that patients who had undergone heart valve surgery were regarded as toothless people unaware of dental care! Some patients even thought that cardiac treatment was primarily based on tooth extraction and hesitated to come through the door of the consulting room for fear of coming out toothless!

Things have rapidly changed with regard to recommendations in prophylactic antibiotic therapy in the 1980s and have been maintained since. Tooth extraction has become commonplace in all patients who had undergone heart valve surgery or had congenital cardiopathy, resulting in the wide use of prescription antibiotics.

New recommendations appeared in 2015. They are based on a better knowledge of pathophysiology and on the benefit-risk ratio of this preventive antibiotic therapy.

Bacteria that cause bacteremia potentially implicated in endocarditis are present in extremely low numbers during mastication and brushing. There was no formal link between dental care and endocarditis occurrence. There has also been no evidence of a reduction in the prevalence of endocarditis with broad antibiotic therapy, which increases the risk of anaphylaxis and the emergence of resistant bacterial strains.

Four major studies, one French², one English ${ }^{8}$, and two American ${ }^{1,5}$, did not find any increase in the incidence of endocarditis following the new recommendations.
Because of the inflammatory reaction and the potential infection that it generates, orthodontics is not exempt from these new recommendations.

\section{Recommendations according to Habib et al. ${ }^{3}$}

Antibiotic therapy should be reserved for interventions involving the oral or gingival mucosa or the periapical dental area.

Antibiotic therapy is not recommended for any other intervention including local anesthetic injections, treatments of superficial caries, or removable orthodontic appliances.

Antibiotic therapy should be reserved for patients at a high risk of endocarditis:

- those with biological or mechanical heart valve prostheses, transcatheter aortic valve replacement, or a prosthetic material used for valvuloplasty (for example, mitral clip)

- those with a history of endocarditis

- those with certain congenital cardiopathies such as cyanogenic cardiopathies (Fallot tetralogy, transposition of the large vessels, Ebstein's anomaly), surgical repair, or transcatheters with prosthetic equipment (interatrial communication closure for example) for up to 6 months after a procedure or for life, in case of residual shunt or valvular regurgitation.

Antibiotic therapy is not recommended in all other cases of valvulopathy or congenital heart disease.

\section{Practical conditions for antibiotic therapy}

Antibiotic therapy, which is reserved only for patients at high risk and administered prior to dental treatments 
involving the oral or gingival mucosa or the periapical dental region, is usually perorally (PO) administered as follows: $2 \mathrm{~g}$ of amoxicillin $(50 \mathrm{mg} / \mathrm{kg}$ for children) an hour before the procedure.

An intravenous injection (at the same dose) is also possible. In case of allergy to beta-lactams, a possible alternative is $600 \mathrm{mg}$ clindamycin PO $(20 \mathrm{mg} /$ $\mathrm{kg}$ for children).

\section{What other preventive measures exist?}

Dental hygiene is paramount with twice-daily brushing and an annual dental consultation (semiannually for high-risk patients) to detect possible caries or other infectious foci.

Any infectious oral (or other) outbreak identified prior to a scheduled cardiac valve operation must be eradicated with a convalescence time of at least 15 days before the valvular intervention. It goes without saying that you must pay attention to rigorous asepsis during all invasive procedures that do not require antibiotic prophylaxis.

Orthodontic treatments do not always lead to antibiotic usage. In case of doubt, consultations between orthodontists and cardiologists are necessary.

\section{HEMORRHAGE}

The other risk of any intervention in the oral sphere is the risk of hemorrhage. The prescription of antithrombotics, both antiaggregants and anticoagulants, has become very common; hence, guidelines are needed in the field of odontology?

\section{Antiplatelet agents}

Since the large Anglo-Saxon studies in the 1980s, antiaggregants have been shown to have a beneficial effect on atherosclerotic disease; they affect the carotid coronary arteries or the lower limbs. They are common after any revascularization, whether surgical (bypass) or instrumental (angioplasty, stent).

Even in asymptomatic patients (such as those with asymptomatic carotid stenosis), they may reduce the risk of vascular events. This prescription has become so common that many patients forget to mention it in the list of their usual treatment!

In stable coronary patients, and also in patients with biological valvular prostheses, the prescription of an antiaggregant is generally limited to monotherapy (acetylsalicylic acid, Aspirin ${ }^{\circledR}$ ) that patients receive indefinitely. The usual dosage is $75 \mathrm{mg} /$ day, which is the dosage that has the best efficacy/ tolerance ratio (usually taken orally). Neurologists prescribe slightly little higher dosages (up to $325 \mathrm{mg} /$ day) in case of ischemic stroke patients.

Sometimes, it can be combination therapy with the simultaneous prescription of two antiaggregants, generally limited in time. Acetylsalicylic acid/ clopidogrel (Aspirin ${ }^{\circledR} /$ Plavix $^{\circledR}$ ), acetylsalicylic acid/prasugrel (Aspirin ${ }^{\circledR} /$ Efient $^{\circledR}$ ), or acetylsalicylic acid/ticagrelor (Aspirin $^{\circledR} /$ Brilique $^{\circledR}$ ) are used for up to 1 year 
after acute coronary syndrome, with or without stenting. After more than a year, the excessive hemorrhagic risk limits the benefit of combination therapy.

\section{Anticoagulants}

The other large antithrombotic family is made up of anticoagulants. An old drug family, they were previously antivitamin $\mathrm{K}$ (AVK). They are three of three types: acenocoumarol (Sintrom $^{\circledR}$ ), fluindione (Previscan ${ }^{\circledR}$ ), and warfarin (Coumadine ${ }^{\circledR}$ ). They require regular biological monitoring, at least monthly, by testing one of the indicators of blood clotting: the international normalized ratio. Over the past 5 years, new anticoagulant treatments have emerged, also known as direct anticoagulants. Three are currently commercialized: dabigatran (Pradaxa ${ }^{\circledR}$ ), rivaroxaban (Xarelto ${ }^{\circledR}$ ), and apixaban (Eliquis ${ }^{\circledR}$ ). They are prescribed at a fixed dose and do not require biological monitoring, except for monitoring of the renal tolerance every 4 to 6 months. However, these new direct anticoagulants have no antidotes in case of drug overdose (as vitamin $\mathrm{K}$ is the antidote for AVK). This is no longer completely true as Pradaxa $^{\circledR}$ has recently been paired with idarucizumab (Praxbind ${ }^{\circledR}$ ), which is a specific antidote. The others should have an antidote soon.

Direct anticoagulants are considered to be more effective than traditional AVKs because their dose is fixed and anticoagulation ability varies less. In addition, they cause less bleeding, particularly intracranial bleeding. They are contraindicated in patients with cardiac mechanical prostheses.
They may be prescribed in venous thromboembolic disease patients, either in the preventive (after surgery) or curative (phlebitis, pulmonary embolism) phase, and atrial fibrillation patients (nonvalvular) to avoid embolic risk (particularly ischemic cerebral accident). They are widely used by cardiologists mainly in case of patients with embolic risk.

In recent years, new predictive scores of ischemic stroke in episodes of atrial fibrillation have been proposed (CHADS score, CHADS Vasc) with a consequent increase in the prescriptions of anticoagulants.

Antithrombotic combinations are also sometimes used. Consider the case of a patient with a mechanical heart valve (lifetime AVK treatment) that caused acute coronary syndrome. A combination of $A V K /$ Aspirin ${ }^{\circledR} /$ Plavix $^{\circledR}$ will was prescribed for a short period (one to three months); then, AVK/Aspirin ${ }^{\circledR}$ was prescribed for 1 year or indefinitely.

It can be seen that cardiologists are not always appreciated by surgeons and their anesthetist (and especially by their patient!). This is also true for oral surgeons, whatever their specialization.

Reaching a consensus is important, and recognition must be given to the working group of the French Society of Oral Surgery for having clearly stated the new recommendations for patients treated with antithrombotics in oral surgery in 2015?. These recommendations need to be updated again with the arrival of new treatments.

These recommendations are in favor of simplifying the management of antithrombotic agents in case of lowrisk hemorrhagic intervention. 
Orthodontic treatments are considered to have low hemorrhagic risk. The discontinuation of anticoagulants (AVK or direct anticoagulants) and antiaggregants (alone or in combination) is not appropriate for all orthodontic treatments.

\section{CONCLUSION}

The latest recommendations are in favor of a reduction in the dosage in prophylactic antibiotic therapy and simplification in the management of antithrombotic treatments.

The field of orthodontics is less exposed to infection and bleeding than other dental fields.

Nevertheless, questioning patients or their family to obtain their prior medical history and providing treatment along with maintaining rigorous asepsis when providing care remain imperative.

In case of doubt, orthodontists/cardiologists can be consulted.

Conflict of interest: The authors declare there is no conflict of interest.

\section{BIBLIOGRAPHY}

1. Desimone DC, et al. Incidence of infective endocarditis caused by viridans group streptococci before and after publication of the 2007 American Heart Association's endocarditis prevention guidelines. Circulation 2012;3;126(1):60-64.

2. Duval $X$, et al. Temporal trends in infective endocarditis in the context of prophylaxis guideline modifications: three successive population-based surveys. J Am Coll Cardiol 2012;59(22):1968-1976.

3. Habib G, Lancellotti P, lung B. 2015 ESC Guidelines on the management of infective endocarditis: a big step forward for an old disease. Heart 2016;102(13):992-994.

4. Mylonakis E, Calderwood SB. Infective endocarditis in adults. N Engl J Med 2001;345(18):1318-330.

5. Pasquali SK, et al. Trends in endocarditis hospitalizations at US children's hospitals: impact of the 2007 American Heart Association Antibiotic Prophylaxis Guidelines. Am Heart J 2012;163(5):894-899.

6. Rosan B, Lamont RJ. Dental plaque formation. Microbes Infect Inst Pasteur 2000;2(13):1599-1607.

7. Société française de chirurgie orale. Gestion péri-opé-ratoire des patients traités par anti-thrombotiques en chirurgie orale, 2015.

8. Thornhill MH, et al. Impact of the NICE guideline recommending cessation of antibiotic prophylaxis for prevention of infective endocarditis: before and after study. BMJ $2011 ; 342:$ d2392. 